\title{
Novedades en UNE ISO 30301 (2019). Sistemas de gestión para los documentos. Requisitos
}

\section{New in UNE ISO 30301 (2019). Management systems for documents. Requirements}

\author{
Carlota Bustelo Ruesta \\ carlota@carlotabustelo.com \\ Presidenta CTN50/SC1. Gestión documentos y aplicaciones \\ Convenor ISO TC46/SC11/WG8. Management system for records
}

\section{Resumen}

Se analiza la actualización de la norma ISO 30301 sobre los requisitos para un sistema de gestión de documentos electrónicos. La norma es más visible debido a que es certificable, como lo demuestran las empresas que la han adoptado.

Palabras clave

ISO 30301; Sistemas de gestión; Documentos electrónicos

\begin{abstract}
The update of the ISO 30301 standard on the requirements for an electronic document management system is analyzed. The norm is more visible because it is certifiable, as evidenced by the companies that have adopted it.
\end{abstract}

Keywords

ISO 30301; Management systems; Electronic documents 
Recibido: 10/12/2019

Aceptado: 23/12/2019

DOI: https://dx.doi.org/10.5557/IIMEI10-N19-028034

Descripción propuesta: Bustelo, Carlota. Novedades en UNE ISO 30301 (2019). Sistemas de gestión para los documentos. Requisitos, 2019. Métodos de Información, 10(19), 28-34

\section{Introducción}

El pasado mes de noviembre se publicó la nueva edición de la norma UNE ISO 30301: 2019 Sistemas de gestión para los documentos. Requisitos (UNE 2019), que es el equivalente idéntico de la norma ISO que se publicó en el mes de febrero del mismo año.

Esta norma sustituye a la norma publicada en el año 2011 (UNE 2011). En estos ocho años, la experiencia en la aplicación de la norma, así cómo el proceso de confluencia con las otras normas de sistemas de gestión, se han traducido en mejoras en el texto y en la organización de conceptos (Wiggins, 2014). Las primeras reacciones recibidas (Kelechava, 2019) sobre la nueva edición son muy positivas, especialmente en el entendimiento de los requisitos, que permiten un mejor funcionamiento de la implantación y de los procesos de auditoría sobre la misma.

\section{La experiencia en la implantación de la norma}

Las normas de sistemas de gestión son instrumentos de la alta dirección da las organizaciones para dirigir las mismas en base a un apolítica y unos objetivos. Sin embargo, cómo pasa con otras normas de sistemas de gestión, son los profesionales de cada área los que proponen, apuestan y trabajan en la implantación de estas normas. En la mayor parte de las organizaciones que han implantado la norma nos encontramos con profesionales de la gestión de documentos que trabajando conjuntamente con la alta dirección han llevado a cabo la implantación. 
No siempre estas implantaciones acaban en una certificación externa, que es lo que hace la norma más visible, pero ya vamos conociendo algunos casos (no hay un registro oficial conjunto de entidades certificadas) que curiosamente no responden a patrones comunes. Sin salir de nuestras fronteras, nos encontramos Universidades Públicas como la Universitat de Lleida, La Universidad Pública de Navarra (UC3M, 2019) o la Escola Superior de Arxivistica y Gestió Documental ESAGED (Alberch et al., 2014); empresas privadas como IECISA, Magma Cultura o Servicios de digitalización y catalogación SL (SDC), y organismos públicos cómo la Autoridad Portuaria de Baleares (Palma, 2016 y APB, 2019). Estas organizaciones ya certificadas según la edición de 2011 tienen dos años para adaptarse a la nueva edición, pero seguramente muchas hagan el recorrido antes ya que la edición de 2019 presenta muchas ventajas con respecto a la anterior, y la transición será bastante fácil.

Uno de los puntos siempre reclamados por las personas que se adentran en la implantación de la norma 30301, es su relación con la norma UNE ISO 15489:2016 Gestión de documentos Conceptos y principios (UNE,2016). En este sentido, la nueva versión presenta la novedad de estar totalmente alineada con la estructura y conceptos de la norma 15489, para que la relación que siempre existió sea cada vez más patente y transparente. De esta forma:

La identificación y valoración, elemento clave de la norma ISO 15489, se encuentra entre los requisitos del capítulo 4.1 - Compresión de la organización y su contexto, con un sub capítulo 4.1.2 Requisitos de gestión de documentos. Estos requisitos se entienden en una doble vertiente, los documentos que la organización debe crear, capturar y gestionar y también los requisitos de cómo debe crearlos, capturarlos y gestionarlos.

La organización de los requisitos operativos de gestión documental que se describen en el Anexo A de la norma 30301 siguiendo los mismos nombres de los capítulos/subcapítulos de la ISO 15489:

- Requisitos relacionados con los procesos de gestión de documentos. Estos procesos que se realizan cuando se opera con los documentos son: la creación, la captura, la clasificación y la indización, el 
almacenamiento, el uso y la reutilización, la conversión y la migración, y la disposición.

- Requisitos relacionados con los instrumentos de gestión de documentos. Estos instrumentos se utilizan para llevar a cabo los procesos mencionados anteriormente. Los instrumentos sobre los que se establecen requisitos son: los cuadros de clasificación, los esquemas de metadatos, las reglas de accesos y permisos y los calendarios de conservación.

- Requisitos relacionados con las aplicaciones de gestión documental, que se utilizan para gestionar los documentos y llevar a cabo los procesos de gestión de documentos.

Todos los requisitos del Anexo A de la norma ISO 30301, incluyen la referencia al requisito anterior para facilitar la implantación de esta versión de la norma a las organizaciones que ya han implantado la versión anterior. Sólo existen dos requisitos nuevos: uno se refiere a cómo gestionar versiones de documentos que así lo requieren, y otro referido a las condiciones que deben cumplirse en procesos de conversión y migración de documentos electrónicos.

\section{La confluencia con otras normas de sistemas de gestión}

La estructura y el texto de los requisitos comunes a todas las normas de sistemas de gestión (que ahora se encuentra en el anexo L de las directivas de ISO) ha sido completamente alineado con la traducción consensuada al español. Dado que la anterior versión de la ISO 30301 (2011) se publicó antes de la inclusión en las Directivas de ISO (ISO/IEC, 2019) del texto consolidado común a todas las normas de sistemas de gestión, había algunos párrafos que debían ser modificados. También posteriormente a su publicación como UNE se consensuó con otros países Latinoamericanos cómo se traduciría ese texto al español y existían algunas traducciones a modificar. El resultado es que en esta versión se expresan los requisitos exactamente de la misma forma que las versiones actuales de normas como la UNE ISO 9001 (UNE, 2015), 14001 (UNE, 2015) o 27001 (UNE, 2017). 
Esto debe facilitar enormemente la implantación conjunta con otras normas de sistemas de gestión, un escenario muy común entre las organizaciones que han implantado o planean implantar la norma.

Los efectos de estos cambios se han traducido en primer lugar, siguiendo la el mismo camino que las demás normas de gestión, en una norma menos prescriptiva y más flexible. Los requisitos exigibles en una auditoría se han reducido en una cuarta parte (de 129 a 98 requisito). Las normas de sistemas de gestión son ahora mucho menos rígidas en la existencia de procedimientos documentados, entendiendo que las organizaciones determinan el tipo de documentación a generar por el propio sistema de gestión y la forma que va a adoptar para ser útil. La idea es acabar con la mala práctica que confunde la implantación de una norma de sistema de gestión con la redacción de la documentación del sistema.

\section{4. ¿Cómo queda la serie de normas UNE ISO 30300?}

La UNE ISO 30301 se incluye en una serie de normas sobre los Sistemas de gestión para los documentos que aún no han sido actualizadas, y que, por lo tanto, todavía siguen vigentes.

- La norma UNE ISO 30300: 2011. Sistemas de gestión para los documentos. Conceptos y vocabulario (UNE, 2011), cuyas definiciones siguen aplicando al entendimiento de la ISO 30301, aunque esta incluya en su propio texto las definiciones comunes a todos los sistemas de gestión. Esta norma se prevé actualizar a mediados del año 2020 su utilización a todas las normas y productos sobre gestión de documentos.

- La norma UNE ISO 30302: 2015). Sistemas de gestión para los documentos. Directrices para la implantación (UNE, 2015, que puede seguir siendo utilizada cómo una guía para la implantación de la versión de 2019 de la 30301, teniendo en cuenta las novedades anteriormente expuestas. Esta norma se adaptará a la nueva versión en los próximos dos años. 


\section{Bibliografía}

ALBERCH, Ramon; CASADESÚS, Anahí; MAURI, Alfred; PERPINYÀ, Remei, 2014. ISO30301 Certification for the Graduate School of Archive and Records Management: a pioneering initiative. En: Archives and Cultural Industries, 2nd Annual Conference ICA; 9th European Conference on Archives; 13th Image and Research Seminar. Girona: Arxius i Indústries Culturals [Consulta: 30 diciembre 2019]. Disponible en : https://www.revistatransformaciondigital.com/2016/04/21/lagestion-documental-digital-la-base-la-transformacion-digital-ejemplo-seguirproyecto-del-sistemagestion/en: http://www.girona.cat/web/ica2014/ponents/textos/id30-ENG.pdf

AUTORIDAD PORTUARIA DE BALEARES, 2019. La autoridad portuaria de baleares certifica su sistema de gestión documental con el referencial ISO 30301. APB[Consulta: 30 diciembre 2019]. Disponible en: http:/ /www.portsdebalears.com/es/noticia/la-autoridad-portuaria-de-balearescertifica-su-sistema-de-gesti $\%$ C3\%B3n-documental-con-el

I

SO/IEC, 2019. ISO/IEC Directives Part 1 and Consolidated ISO Supplement. [Consulta: 30 diciembre 2019]. Disponible en: https://www.iso.org/directives-andpolicies.html

KELECHAVA, Brad, 2019. ISO 30301:2019 - Management Systems For Records (MSR) Requirements. En: American National Standrds Institute, 2019. [Consulta: 30 diciembre 2019]. Disponible en: https://blog.ansi.org/2019/03/iso30301-2019-management-records-requirement/\#gref

PALMA VILLALÓN, María del Valle, 2016. La gestión documental es la base de la transformación digital. Un ejemplo a seguir: el proyecto SGD conforme a la ISO 30301 de la Autoridad Portuaria de Baleares. En: Revista Transformación Digital | jueves 21 - abril - 2016. [Consulta: 30 diciembre 2019]. Disponible en: https://www.revistatransformaciondigital.com/2016/04/21/la-gestiondocumental-digital-la-base-la-transformacion-digital-ejemplo-seguir-proyecto-delsistema-gestion/

UNE, 2011. UNE ISO 30300:2011 Sistemas de gestión para los documentos. Fundamento y vocabulario. Madrid: UNE 
UNE, 2011. UNE ISO 30301:2011 Sistemas de gestión para los documentos. Requisitos Madrid: UNE

UNE, 2015. UNE ISO 14001:2015 Sistemas de gestión de la calidad. Requisitos. Madrid: UNE

UNE, 2015. UNE ISO 30302:20151 Sistemas de gestión para los documentos. Guia de implementación. Madrid: UNE

UNE, 2015. UNE ISO 9001:2015 Sistemas de gestion de la calidad. Requisitos. Madrid: UNE

UNE, 2016. UNE ISO 15489:2016 Gestión de documentos Conceptos y principios. Madrid: UNE

UNE, 2017. UNE-EN ISO/IEC 27001:2017. Tecnología de la información. Técnicas de seguridad. Sistemas de Gestión de la Seguridad de la Información. Requisitos. Madrid: UNE

UNE, 2019. UNE ISO 30301:2019 Sistemas de gestión para los documentos. Madrid: UNE

UNIVERSIDAD CARLOS III DE MADRI, 2019.. Seminario sobre la implantación de un sistema de gestión para los documentos (SGD) en la Universidad. La aportación de UNE ISO 30301 y UNE ISO 15489, celebrado el 4 de abril de 2019 en el Campus de Puerta de Toledo (Madrid) de la Universidad Carlos III de Madrid. Madrid: Universidad Carlos III de Madrid [Consulta: 30 diciembre 2019]. Disponible en: https://media.uc3m.es/video/5cac85cc8f420851908b4575 